\title{
Estrutura Fatorial da Versão Portuguesa da Escala de Narcisismo Hipersensível
}

\author{
Factor Structure of the Portuguese Version of the Hypersensitive Narcissism Scale
}

\author{
Cândida Pereira ${ }^{1}$ e Rui Paixão ${ }^{2}$
}

\begin{abstract}
Resumo
O narcisismo apresenta-se como um fenómeno multidimensional, com dois padrões: grandioso e vulnerável. A escala de narcisismo hipersensível (HSNS), objeto deste estudo, procura avaliar o narcisismo vulnerável. Os estudos foram realizados com uma amostra de 273 sujeitos da população geral que responderam ao HSNS, a um questionário sociodemográfico, ao Inventário de Personalidade Narcísica e ao NEO-Five Factor Inventory. Os estudos estatísticos exploratórios e confirmatórios evidenciam que o HSNS revela um construto consistente com uma estrutura bifatorial, com um Fator de Egocentrismo e um Fator de Hipersensibilidade ao julgamento. Evidenciam-se algumas fragilidade ao nível da fiabilidade (alfa de Cronbach inferiores a .70), mas as comparações entre as diferentes escalas confirmam que os diferentes padrões de narcisismo apresentam traços de personalidade diferentes, reforçando a necessidade de os analisar de forma distinta.
\end{abstract}

Palavras-chave: HSNS, NPI, NEO-FFI, narcisismo

\begin{abstract}
Narcissism presents itself as a multidimensional phenomenon, with two patterns: grandiose and vulnerable. The Scale of Hypersensitive Narcissism (HSNS), focus of this study, seeks to evaluate vulnerable narcissism. The studies were performed with a sample of 273 subjects from the general population who responded to HSNS, a socio-demographic questionnaire, the Narcissistic Personality Inventory and the NEO-Five Factor Inventory. The exploratory and confirmatory statistical studies show that HSNS reveals a construct consistent with a two-factor structure, with an Egocentrism Factor and a Hypersensitivity to Judgment Factor. Some weaknesses in reliability (Cronbach's alpha below .70) are evident, but comparisons between different scales confirm that different patterns of narcissism have different personality traits, reinforcing the need to analyze them differently.
\end{abstract}

Keywords: HSNS, NPI, NEO-FFI, narcissism

\footnotetext{
${ }^{1}$ Mestre em Psicologia Clínica. Consultora de Recursos Humanos na Elevus e Psicóloga Clínica na Ana Lito - Clínica Dentária de Recardães. Rua da Boiça, n³4 3780-051 Ancas, Anadia, Portugal. Tel.: +351914343423. E-mail: candida—pereira@ hotmail.com

${ }^{2}$ Doutorado em Psicologia Clínica. Professor Associado da Faculdade de Psicologia e de Ciências da Educação da Universidade de Coimbra e Investigador do Centro de Estudos Sociais da Universidade de Coimbra. Rua do Colégio Novo 3001-802 Coimbra, Portugal. Tel.: +351239851450. E-mail: rpaixao@fpce.uc.pt
} 


\section{Introdução}

O narcisismo pode ser considerado sob a perspetiva patológica enraizada na psicologia clínica (Besser \& Zeigler-Hill, 2010; Zeigler-Hill, Green, Arnau, Sisemore, \& Myers, 2011) ou sob uma perspetiva dimensional, como um traço da personalidade (Foster \& Campbell, 2007; Miller \& Campbell, 2008; Zeigler-Hill et al., 2011), havendo mesmo quem considere que o narcisismo tem subjacente uma expressão normal e uma expressão patológica (Roche et al., 2013).

O narcisismo tem sido associado a dois padrões: o narcisismo grandioso e o narcisismo vulnerável $^{1}$ (Dickinson \& Pincus, 2003; Hendin \& Cheek, 1997; Kealy \& Rasmussen, 2012; Miller et al., 2011; Miller, Price, Gentile, Lynam, \& Campbell, 2012; Pincus \& Lukowitsky, 2010; Ronningstam, 2009). Ambos têm como características dominantes a sensibilidade ao desprezo e a autoabsorção (Luchner et al., 2011), o descontrolo da hostilidade e dos impulsos negativos (Wink, 1996) e os sentimentos de direito (feelings of entitlement) (Zeigler-Hill et al., 2011). No entanto, os dois têm sido associados a funcionamentos psicológicos relativamente distintos.

Pessoas com elevados níveis de narcisismo grandioso (NG) estão associadas a fantasias de sucesso, poder sobre os outros, superioridade, perfeição e adulação (Cain, Pincus \& Ansell, 2008; Campbell et al., 2002a; Cooper, 2009; Foster \& Campbell, 2005; Foster, Campbell, \& Twenge, 2003; Kealy, Ogrodniczuk, Joyce, Steinberg, \& Piper, 2013; Kealy \& Rasmussen, 2012; Lee \& Ashton, 2005; Maxwell, Donellan, Hopwood, \& Ackerman, 2011; Miller, Widiger \& Campbell, 2010; Pincus \& Lukowitsky, 2010; Ronnigstam, 2009; Thomaes et al., 2009). Acreditam que merecem um tratamento especial e que estão acima das convenções e normas sociais, colocando-se num pedestal de onde possam obter a admiração dos outros (Kealy \& Rasmussen, 2012; Thomaes et al., 2009). Sobrestimam as suas competências e conquistas, reprimem os aspetos

\footnotetext{
${ }^{1}$ No presente estudo e de modo geral na literatura os termos vulnerável, encoberto e hipersensível surgem como sinónimos, remetendo para o mesmo construto. De modo similar, o narcisismo grandioso poderá também surgir na literatura como aberto.
}

negativos do self, atribuem a culpa por todos os retrocessos ou contratempos a circunstâncias externas, distorcem a informação exterior desconfirmadora das suas fantasias e tentam derrotar quem ameace as suas autorrepresentações (Dickinson \& Pincus, 2003; Kealy \& Rasmussen, 2012; Morf \& Rhodewalt, 2001; Pincus \& Lukowitsky, 2010; Thomaes et al., 2009). Revelam uma faceta manipuladora, arrogante e de exploração do outro, inveja intensa, um estilo interpessoal exibicionista e falta de empatia (Dickinson \& Pincus, 2003; Kealy \& Rasmussen, 2012; Morf \& Rhodewalt, 2001; Pincus \& Lukowitsky, 2010). As diferentes estratégias de autovalidação permitem-lhes manter o Ego inflamado e negar as vulnerabilidades, sendo muito pouco frequentes sentimentos de inferioridade ou depressão. Tendem a denegar o stress emocional e interpessoal e a transparecer uma sensação de equilíbrio e segurança social, autoconfiança e associam-se a vidas de sucesso profissional (Miller et al., 2012; Wink, 1996).

$\mathrm{O}$ narcisismo vulnerável $(\mathrm{NV})$ tem também subjacente fantasias grandiosas, mas apenas se revelam no contacto próximo (Wink, 1996). As pessoas com níveis mais elevados de NV demonstram uma aparente empatia e uma superfície modesta, caraterizada pela timidez, vergonha, insegurança, medo e ansiedade em construir e manter relacionamentos sociais (Besser \& Zeigler-Hill, 2010; Dickinson \& Pincus, 2003; Given-Wilson et al., 2011; Ronningstam, 2009).

As suas dificuldades interpessoais são mediadas pela desregulação do self, pela identidade difusa, autoimagem empobrecida, frágil e tendencialmente negativa, e pela instabilidade emocional. Ou seja, o NV está associado à vivência de emoções extremas e dificuldade em controlar os estados de humor, sobretudo quando as fortes necessidades de admiração e expetativas idealizadas para o self e para os outros não são concretizadas (Ackerman et al., 2011; Dickinson \& Pincus, 2003; GivenWilson et al., 2011; Pincus \& Lukowitsky, 2010; Tritt et al., 2010). Na tentativa de controlar a sua autoestima e de se proteger da desilusão e da vergonha sobre as expectativas não correspondidas, evitam os relacionamentos e adotam comportamentos de inibição e isolamento 
social (Dickinson \& Pincus, 2003; Given-Wilson et al., 2011).

A agressividade tem sido bastante associada ao narcisismo (Miller et al., 2010b) e inclusive existem estudos que relacionam o narcisismo com indicadores criminais (Twenge et al., 2017). Os resultados do estudo de Ryan, Weikel e Sprechini (2008) demonstram que existe uma ligação entre o narcisismo e as atitudes sexuais e a violência doméstica. No entanto, existem estudos que referem que o NV não está associado com comportamentos externalizantes como comportamentos antissociais, agressão ou uso de substâncias (Miller et al., 2010a).

Pensa-se que pessoas com elevados níveis de NG tendem, também, a apresentar elevados níveis de desagradabilidade (falta de modéstia e agressividade) por motivos instrumentais de ganho pessoal e para conseguir estatuto e poder (Miller et al., 2010a). Já a desagradabilidade associada ao NV pensa-se que esteja elencada à desregulação afetiva associada às experiências da primeira infância (Miller et al., 2010a).

O narcisismo surge associado a abusos na infância e/ou negligência (Miller \& Campbell, 2008), mas alguns estudos remetem para esta associação apenas ao nível do NV, nomeadamente com abuso sexual infantil, físico, verbal e emocional, estilos de parentalidade intrusivos, controladores, com pouca afetividade, com pouca supervisão parental ou elevada intromissão psicológica (Miller et al., 2010a; Miller et al., 2010b). Estes estilos de vinculação patológicos falham na educação da criança para a regulação das suas emoções, na tolerância ao stress e frustração e, ao mesmo tempo, encorajam exibições emocionais extremas (Miller et al., 2010a; Miller et al., 2011). Também, pessoas com abusos ou negligência documentada na infância têm maior probabilidade de desenvolver perturbações da personalidade na vida adulta (Johnson et al., 1999) ou de se envolverem e persistirem em crimes e outras problemáticas psicossociais (Basto-Pereira et al., 2016), ainda que não se conheçam bem os mecanismos associados (Lee \& Hoaken, 2007). O estudo do $\mathrm{NV}$ poderá ajudar a compreender esses mecanismos através dos quais o maltrato infantil se associa a consequências na vida adulta.
Por outro lado, alguns autores mencionam que o narcisismo se desenvolve em resultado da sobreproteção e sobrevalorização que os pais fazem das conquistas dos seus filhos e de um reforço que não é contingente a comportamentos atuais, que impede a criança de ter uma imagem real de si, não a permitindo experimentar sentimentos de frustração, falha ou desilusão (Miller \& Campbell, 2008).

O narcisismo (como medido pelo Narcissism Personality Inventory - NPI) surge associado de forma positiva com a autoestima e negativamente relacionado com depressão e tristeza (Miller \& Campbell, 2008; Miller et al., 2010a). Apenas o NV está relacionado com falta de bem-estar, incompetência, desajustamento pessoal e baixa autoestima, revelando-se mais disfuncional do que o NG (Wink, 1996) e associando-se tendencialmente à patologia internalizante (Ackerman et al., 2011). Estas pessoas vivenciam elevados níveis de emoções negativas, associando-se a problemas psicológicos como o stress, ansiedade e depressão (Miller et al., 2010b; Miller et al., 2011). Em contrapartida, o NG habitualmente surge negativamente associado ou não relacionado com estas problemáticas. De modo similar, o NG surge associado ao distúrbio da personalidade antissocial e histriónico, enquanto o NV está associado com os distúrbios da personalidade borderline e evitante (Miller et al., 2011).

O NG (NPI) e a patologia narcísica têm sido associados com maior incidência ao sexo masculino (Corry et al., 2008; Morf \& Rhodewalt, 2001). Por outro lado, o sexo feminino tem sido associado sobretudo à patologia borderline (Morf \& Rhodewalt, 2001), que tem sido associada ao NV (Miller \& Campbell, 2008; Miller et al., 2011; Morf \& Rhodewalt, 2001). Alguns autores chegam mesmo a considerar que o NV pode estar a ser erradamente diagnosticado como perturbação borderline (Dickinson \& Pincus, 2003), o que sugere que o NG pode ser mais prevalente no sexo masculino e o NV no sexo feminino.

Os diferentes tipos de narcisismo encontramse relacionados com diferentes resultados e interações do ponto de vista psicopatológico e psicoterapêutico. As características do NG reduzem a utilidade de tratamentos, enquanto as 
características do NV poderão promover a sua utilização (Miller et al., 2010b; Miller et al., 2011).

Com o passar do tempo, o narcisismo tem um impacto negativo nos relacionamentos amorosos devido à sua tendência para a infidelidade e para ver a relação como um jogo (Miller et al., 2010b). No âmbito das relações íntimas, ambos são vistos como autoritários, intolerantes, cruéis, argumentativos, desonestos, oportunistas e arrogantes. No entanto, os NG são também descritos como agressivos, determinados e de difícil trato (Wink, 1991).

$\mathrm{O}$ reconhecimento das diferenças entre os dois padrões de narcisismo é bastante importante devido às suas associações com diferentes traços básicos da personalidade, comportamentos, fatores etiológicos ambientais, estilos de vinculação, sintomas, psicopatologia e utilização dos recursos clínicos (Miller et al., 2012).

Até 1979 haviam dificuldades na investigação sobre o construto do narcisismo devido à falta de um instrumento de medição apropriado. Raskin e Hall (1979) desenvolveram o Inventário de Personalidade Narcisista (NPI; Narcistic Personality Inventory), que desde então se tornou a medida de narcisismo mais utilizada (Ackerman et al., 2011; Cain et al., 2008; Corry et al. 2008; Kurbarych et. Al., 2004).

Em Portugal o NPI foi submetido a um estudo psicométrico através de uma amostra juvenil forense (Pechorro et al., 2016). No mesmo trabalho foram ainda estudadas as versões reduzidas do NPI-16 e do NPI-13. A versão reduzida de 13 itens de Gentile et al. (2013) foi ainda testada em mais dois trabalhos: um com uma subamostra juvenil forense e uma subamostra de participantes de escolas públicas (Pechorro et al., 2018); e outro com uma amostra proveniente de estabelecimentos públicos de ensino das zonas de Lisboa, Coimbra e Algarve (Pechorro et al., 2018).

No entanto, o foco do NPI no narcisismo grandioso minimiza a sua utilidade para a medição e compreensão dos aspetos multidimensionais do construto (Corry et al., 2008). Estes autores referem ainda que os resultados da sua investigação sugerem a necessidade de reformulação da escala para incluir e distinguir os itens que avaliam o NG e o NV reforçando a necessidade de distinção dos padrões, como referido anteriormente.
Hendin e Cheek (1997) remetem para a Escala de Narcismo de Murray (1938) como um exemplo de uma medida mais associada ao padrão de NV. Considerando que isto tem sido negligenciado, propõem estudar a relação entre as várias medidas de modo a alcançar os aspetos "encobertos" do narcisismo que o NPI não consegue captar adequadamente. A Escala de Narcisismo Hipersensível (HSNS; The Hypersensitive Narcissim Scale; Hendin \& Cheek, 1997) surge, assim, como uma medida que se propõe medir o padrão de narcisismo hipersensível/encoberto que o NPI não capta, dentro do intervalo normal de diferenças individuais. Não obstante, na ausência de dados da medida para a população portuguesa sentiu-se a necessidade de realizar um estudo preliminar do HSNS, pois os testes psicológicos são uma das ferramentas mais importante ao serviço da prática profissional e da investigação em psicologia (Prieto et al., 1999).

O objetivo deste trabalho prende-se com uma análise psicométrica, do HSNS numa amostra da população portuguesa. Serão avaliadas a estrutura factorial da escala, a consistência interna e, considerando o cruzamento entre medidas, a validade de construto e divergente. Neste último caso, a hipótese em estudo prevê baixas correlações do HSNS com a amabilidade e a extroversão e elevadas correlações com o neuroticismo, de acordo com os trabalhos de Hendin e Cheek (1997), Houlcroft et al. (2012), Miller, Dir, Gentile, Wilson e Campbell (2010), Miller et al. (2010b) e Miller et al. (2011). Em oposição, prevêem-se correlações elevadas entre o NPI e a extroversão e baixas correlações com o neuroticismo e a amabilidade (Houlcroft et al., 2012; Miller \& Campbell, 2008; Paulhus \& Williams, 2002; Miller et al., 2010b; Miller et al., 2011).

\section{Método}

\section{Participantes}

Como se pode verificar no Quadro 1 participaram neste projecto 273 sujeitos, maioritariamente do sexo feminino, solteiros, estudantes ou especialistas de atividades intelectuais e científicas. A média de idades é de $23.35 \pm 4.48$ e a escolaridade de $15.57 \pm 2.45$. Os critérios de inclusão foram ter $\geq 18$ anos de idade e ter como língua materna o português. 


\begin{tabular}{|c|c|c|c|c|}
\hline & $\mathrm{n}$ & $\%$ & $\mathrm{M}$ & DP \\
\hline \multicolumn{5}{|l|}{ sexo } \\
\hline feminino & 184 & 67.4 & & \\
\hline masculino & 89 & 32.6 & & \\
\hline idade & & & 23.35 & 4.48 \\
\hline $\begin{array}{l}\text { anos de escolaridade } \\
\text { estado civil }\end{array}$ & & & 15.57 & 2.45 \\
\hline solteiro & 243 & 89 & & \\
\hline união de facto & 17 & 6.2 & & \\
\hline casado & 13 & 4.8 & & \\
\hline ocupação/profissão & & & & \\
\hline $\begin{array}{l}\text { estudantes } \\
\text { especialistas de }\end{array}$ & & 66.1 & & \\
\hline $\begin{array}{l}\text { actividades } \\
\text { intelectuais e } \\
\text { científicas } \\
\text { técnicos e }\end{array}$ & & 20.7 & & \\
\hline $\begin{array}{l}\text { profissionais de } \\
\text { nível intermédio }\end{array}$ & & 4.1 & & \\
\hline desempregados & & 3.7 & & \\
\hline $\begin{array}{l}\text { trabalhadores de } \\
\text { serviços }\end{array}$ & & 3 & & \\
\hline $\begin{array}{l}\text { operadores de } \\
\text { instalações e } \\
\text { máquinas }\end{array}$ & & 0.7 & & \\
\hline $\begin{array}{l}\text { trabalhadores não } \\
\text { qualificados }\end{array}$ & & 0.7 & & \\
\hline
\end{tabular}

\section{Procedimentos}

A técnica de amostragem utilizada foi não probabilística e a amostra recolhida com recurso à rede social "facebook". Nesta plataforma os respondentes eram convidados a participar no projeto, depois de informados sobre os objetivos do estudo, da confidencialidade dos dados e do caráter voluntário da sua colaboração, tendo a possibilidade de desistir em qualquer momento. Para participar todos os respondentes tiveram de declarar previamente ter compreendido os objetivos e aceitar participar livremente, selecionando a respetiva opção. $\mathrm{O}$ acesso aos questionários online, colocados na plataforma "Google forms", realizou-se através de um link específico.

O protocolo incluiu um questionário de recolha de dados sociodemográficos, o HSNS, o Inventário de Personalidade Narcísica (NPI) e o NEO-Five Factor Inventory (NEO-FFI). Estas últimas escalas foram utilizadas nos estudos de validade do HSNS.

A adaptação do HSNS passou por um primeiro processo de tradução-retroversão que seguiu os três passos referidos por Hill e Hill (2005): a tradução do inglês para o português por uma especialista em inglês, de língua materna portuguesa; a retroversão da versão portuguesa para o inglês, por outra especialista em inglês, de língua materna portuguesa. $\mathrm{O}$ estudo comparativo da versão original e da versão retrovertida evidenciou a inexistência de diferenças significativas. A versão portuguesa assim obtida foi, ainda, sujeita a uma fase pré-teste com 10 sujeitos.

Ao nível dos procedimentos estatísticos foram realizadas análises de consistência interna, análise fatorial exploratória (AFE) e análise fatorial confirmatória (AFC), testando o modelo unidimensional proposto por Hendin e Cheek (1997) e a solução obtida com a AFE. A solução final obtida para o HSNS foi ainda correlacionada com o NEO-FFI.

\section{Instrumentos}

- Escala de Narcisismo Hipersensível (HSNS; The Hypersensitive Narcissim Scale, Hendin \& Cheek, 2013).

Este instrumento de autorresposta é composto por 10 itens que pretendem avaliar a hipersensibilidade e a vulnerabilidade associada ao narcisismo, mais especificamente ao narcisismo vulnerável em populações não clínicas (Hendin \& Cheek, 1997), através de uma escala do tipo de Likert de 1 (nada verdadeiro para mim) a 5 (muito verdadeiro para mim), podendo os resultados variar entre 10 e 50 . Valores mais elevados estão associados à maior presença do construto no funcionamento do sujeito (Arble, 2008). A versão original (Hendin \& Cheek, 1997), revela uma estrutura unifatorial associada a um alfa de Cronbach entre .62 e .76. Na versão italiana de Fossati et al. (2009) é adotada uma estrutura bifatorial, que associam à hipótese de um fator de segunda ordem (não testado). Nesta versão (Fossati et al., 2009), os alfas são muito baixos: a escala completa revela um $\alpha=.69$, a componente hipersensibilidade ao julgamento (itens $1,2,3,6,7,9$ ) demonstra um $\alpha=.66$ e a componente egocentrismo (itens $4,5,8,10$ ) um $\alpha=$.62. O estudo de Ripoll, Salazar e Bobes (2010), com a população espanhola, revela uma estrutura de três fatores, com alfa de Cronbach de .78 e .73 . O fator egoísmo incluindo os itens 4,5 e 10; o fator hipersensibilidade é representado pelos itens 2, 3, 6 e 7; e, finalmente, o autoabsorção ("ensimismamiento") com os itens 1, 8 e 9. 
- Inventário de Personalidade Narcísica (NPI; Narcistic Personality Inventory, Raskin \& Hall, 1979; Raskin \& Terry, 1988; Pereira, 2015).

O NPI é um instrumento de autorresposta, composto por 40 pares de itens dicotómicos em que uma opção é considerada narcísica (correspondendo a 1 ponto) e outra não narcísica (0 pontos). Os resultados totais podem variar entre 0 e 40. O estudo da escala revelou uma média de 15.55 \pm 6.66 (Raskin \& Terry, 1988). Várias questões têm sido levantadas relativamente ao construto da medida, havendo diversas propostas de estruturas fatoriais (Ackerman et al., 2011; Corry et al., 2008; Emmons, 1987; Raskin \& Terry, 1988; Kurbarych, Deary, \& Austin, 2004). Não obstante, o NPI tem sido a "medida de ouro" do narcisismo grandioso na população não clínica (Corry et al., 2008).

No presente estudo, a versão utilizada é composta por 12 dos pares de itens dicotómicos em que uma opção é considerada narcísica (correspondendo a 1 ponto) e outra não narcísica (0 pontos). Apresenta uma estrutura hierárquica, com um fator de segunda ordem (NPI total), e 3 fatores: o fator liderança/autoridade (itens 1, 33, $10,12)$ demonstra um $\alpha=.67$, o fator vaidade (itens $29,19,15,26)$ um $\alpha=.64$ e o fator pessoa especial (itens $40,9,39,36$ ) um $\alpha=62$. Os 12 itens na totalidade revelam um $\alpha=.72$ (Pereira, 2015).

- NEO-Five Factor Inventory (NEO-FFI; Costa \& McCrae, 1989; versão portuguesa de Lima \& Simões, 2000).

Este instrumento de autorresposta é uma versão curta do NEO-Personality InventoryRevised, composta por 60 itens, que pretendem avaliar os cinco fatores básicos da personalidade: Neuroticismo, Extroversão, Abertura à Experiência, Amabilidade e Conscienciosidade, através de uma escala de tipo Likert de 5 pontos entre 0 (discordo fortemente) e 4 (concordo fortemente). A consistência interna do NEO-FFI apresenta alfas de Cronbach que variam entre .68 e .86 na versão original (Magalhães, et al., 2014; Pedroso-Lima et al.,2014). Na versão portuguesa a consistência interna dos fatores demonstra ser adequada: conscienciosidade $\quad(\alpha=81)$, neuroticismo $(\alpha=.81)$, extroversão $(\alpha=.75)$, amabilidade $(\alpha=.72)$ e abertura à experiência $(\alpha=.71)$ (Lima et al., 2014; Magalhães et al.,
2014). Na presente amostra a consistência interna assume valores diferentes: conscienciosidade $(\alpha=.86), \quad$ neuroticismo $\quad(\alpha=.87), \quad$ extroversão $(\alpha=.74)$, amabilidade $(\alpha=.48)$ e abertura à experiência $(\alpha=.67)$.

\section{Resultados}

Os 10 itens do HSNS foram submetidos a uma Análise Fatorial Exploratória (AFE), através do método de Fatorialização do Eixo Principal, com retenção de fatores mediante o critério de Kaiser. Foi aplicada a rotação promax, seguindo o pressuposto teórico de que os fatores estão relacionados entre si. A solução rodada revela uma estrutura de dois fatores, com uma correlação entre os fatores de .44 .

Os itens $4,5,8$ e 10 representam o fator 1 (egocentrismo) e os itens 1, 2, 3, 7 e 9 o fator 2 (hipersensibilidade ao julgamento). $\mathrm{O}$ item 6 revela uma saturação inferior a .32 , tendo sido retirado e repetida a AFE (Quadro 2).

A Análise Fatorial Confirmatória (AFC) foi realizada com o objetivo de verificar qual o modelo com melhor ajustamento. Para o efeito testou-se o Modelo Unifatorial (Hendin \& Cheek, 1997) e o modelo de dois fatores obtido na $\mathrm{AFE}^{2}$. Os modelos testados revelam um mau ajustamento inicial, tendo sido realizados ajustamentos para a sua melhoria. No Quadro 3 podem observar-se os resultados dos modelos iniciais e dos modelos com as respetivas alterações (passo-a-passo).

$\mathrm{O}$ modelo de dois fatores com 8 itens (exclusão dos itens 1 e 6) e 2 covariâncias entre erros (Modelo 2F, Figura 1), apresenta valores de ajustamento global adequados com um Rácio $\chi^{2} / g .1$. inferior a 2, qui-quadrado não significativo, valores de GFI e CFI superiores a .95 e valor de RMSEA inferior a .06. Ao nível do ajustamento local todos os itens demonstram valores de coeficientes de regressão adequados (entre $.36 \mathrm{e}$ .64; $p<.001)$ (Tabachnick \& Fidel, 2007).

\footnotetext{
${ }^{2} \mathrm{Na}$ sequência da hipótese de Fossati et al. (2009), foi ainda considerada a possibilidade de um fator de segunda ordem, para o modelo de dois fatores obtido na AFE, mas a escala não apresenta as condições necessárias. $\mathrm{O}$ número de graus de liberdade para a equação de regressão deste fator é inferior aos parâmetros a estimar, por isso, o modelo não é identificável (é subestimado) (Tabachnich \& Fidel, 2007).
} 
Quadro 2. Resultados da AFE, através do método da fatorialização do eixo principal, com rotação promax, da Escala de Narcisismo Hipersensível (HSNS) N=273

\begin{tabular}{|c|c|c|c|}
\hline & $\begin{array}{l}\text { Fator } 1 \\
\text { Egocentrismo }\end{array}$ & $\begin{array}{c}\text { Fator } 2 \\
\text { Hipersensibilidade } \\
\text { ao Julgamento }\end{array}$ & $\mathrm{h}^{2}$ \\
\hline $\begin{array}{l}10 \text { - Eu aborreço-me ou irrito-me em segredo quando as outras } \\
\text { pessoas vêm até mim com os seus problemas, pedir o meu } \\
\text { tempo e simpatia. }\end{array}$ & .682 & -.193 & .386 \\
\hline $\begin{array}{l}5 \text { - Sinto que "já tenho em mãos" o suficiente sem me } \\
\text { preocupar com os problemas dos outros. }\end{array}$ & .576 & -.089 & .295 \\
\hline $\begin{array}{l}8 \text { - Eu fico facilmente envolvido nos meus interesses e esqueço- } \\
\text { me da existência dos outros. }\end{array}$ & .514 & .111 & .327 \\
\hline $\begin{array}{l}4 \text { - Eu não gosto de dividir os créditos de um sucesso com os } \\
\text { outros. }\end{array}$ & .349 & .261 & .271 \\
\hline $\begin{array}{l}7 \text { - Frequentemente interpreto os comentários dos outros de } \\
\text { forma pessoal. }\end{array}$ & .021 & .551 & .314 \\
\hline $\begin{array}{l}2 \text { - Os meus sentimentos são facilmente magoados pela } \\
\text { ridicularização e pelo desprezo dos outros. }\end{array}$ & -.101 & .495 & .211 \\
\hline $\begin{array}{l}3 \text { - Quando entro numa sala torno-me, frequentemente, auto } \\
\text { consciente e sinto que os olhares dos outros estão virados para } \\
\text { mim. }\end{array}$ & .120 & .437 & .252 \\
\hline $\begin{array}{l}1 \text { - Sou capaz de ficar inteiramente absorvido nos meus } \\
\text { pensamentos acerca dos meus assuntos pessoais, da minha } \\
\text { saúde, das minhas preocupações ou relações com os outros. }\end{array}$ & -.189 & .350 & .100 \\
\hline $\begin{array}{l}9 \text { - Eu não gosto de estar num grupo, a não ser que eu saiba que } \\
\text { sou apreciado por, pelo menos, um dos presentes. }\end{array}$ & .199 & .348 & .222 \\
\hline \% Variância explicada & $18.93 \%$ & $7.49 \%$ & \\
\hline \% Variância explicada total & \multicolumn{2}{|r|}{$26.42 \%$} & \\
\hline
\end{tabular}

Quadro 3. Resultados nos diferentes estudos da AFC da Escala de Narcisismo Hipersensível (HSNS)

\begin{tabular}{|c|c|c|c|c|c|c|c|c|}
\hline Modelo & $\chi^{2}($ g.l) & $\chi^{2} / g .1$. & $p$. & GFI & CFI & RMSEA & AIC & CAIC \\
\hline $\begin{array}{l}\text { Modelo Unifatorial } \\
\text { (Hendin \& Cheek, 1997) }\end{array}$ & 96.02 (35) & 2.74 & $<.001$ & .93 & .78 & .08 & 136.02 & 228.21 \\
\hline $\begin{array}{l}\text { Modelo Unifatorial } \\
\text { (Hendin \& Cheek, 1997) } \\
9 \text { covariância entre erros }\end{array}$ & $29.47(26)$ & 1.13 & .290 & .98 & .99 & .02 & 87.47 & 221.14 \\
\hline $\begin{array}{l}\text { Modelo Unifatorial } \\
\text { (Hendin \& Cheek, 1997) } \\
11 \text { covariância entre erros }\end{array}$ & $17.64(24)$ & .735 & .820 & .99 & 1 & .00 & 79.64 & 222.53 \\
\hline $\begin{array}{l}\text { Modelo Unifatorial } \\
\text { (Hendin \& Cheek, 1997) } \\
8 \text { Itens } \\
\text { (exclusão itens } 1 \text { e 2) }\end{array}$ & $60.68(20)$ & 3.03 & $<.001$ & .95 & .84 & .08 & 92.67 & 166.43 \\
\hline $\begin{array}{l}\text { Modelo Unifatorial } \\
\text { (Hendin \& Cheek, 1997) } \\
8 \text { Itens } \\
\text { (exclusão itens } 1 \text { e 2) } \\
7 \text { covariância entre erros }\end{array}$ & $14.05(13)$ & 1.08 & .370 & .99 & 1 & .02 & 60.05 & 166.08 \\
\hline $\begin{array}{l}\text { Modelo Unifatorial } \\
\text { (Hendin \& Cheek, 1997) } \\
7 \text { itens } \\
\text { (exclusão itens } 1,2 \text { e 5) }\end{array}$ & 30.91 (14) & 2.21 & .006 & .97 & .91 & .07 & 58.91 & 123.44 \\
\hline $\begin{array}{l}\text { Modelo com } 2 \text { fatores } \\
\text { (Hendin \& Cheek, 1997) } \\
7 \text { itens } \\
\text { (exclusão itens } 1.2 \text { e } 5 \text { ) } \\
5 \text { covariâncias entre erros }\end{array}$ & $7.25(9)$ & .806 & .611 & .99 & 1 & .00 & 45.25 & 132.83 \\
\hline $\begin{array}{l}\text { Modelo com } 2 \text { fatores } \\
\text { (9 itens - exclusão item 6) } \\
\text { Modelo com } 2 \text { fatores }\end{array}$ & $61.60(26)$ & 2.37 & $<.001$ & .95 & .86 & .07 & 99.60 & 187.18 \\
\hline $\begin{array}{l}8 \text { Itens } \\
\text { (exclusão itens } 1 \text { e 6) } \\
\text { Modelo 2F }\end{array}$ & $44.71(19)$ & 2.35 & .001 & .96 & .89 & .07 & 78.71 & 157.07 \\
\hline $\begin{array}{l}8 \text { Itens } \\
\text { (exclusão itens } 1 \text { e } 6 \text { ) } \\
2 \text { covariâncias entre erros }\end{array}$ & 24.69 (17) & 1.45 & .102 & .98 & .97 & .04 & 62.68 & 150.26 \\
\hline
\end{tabular}




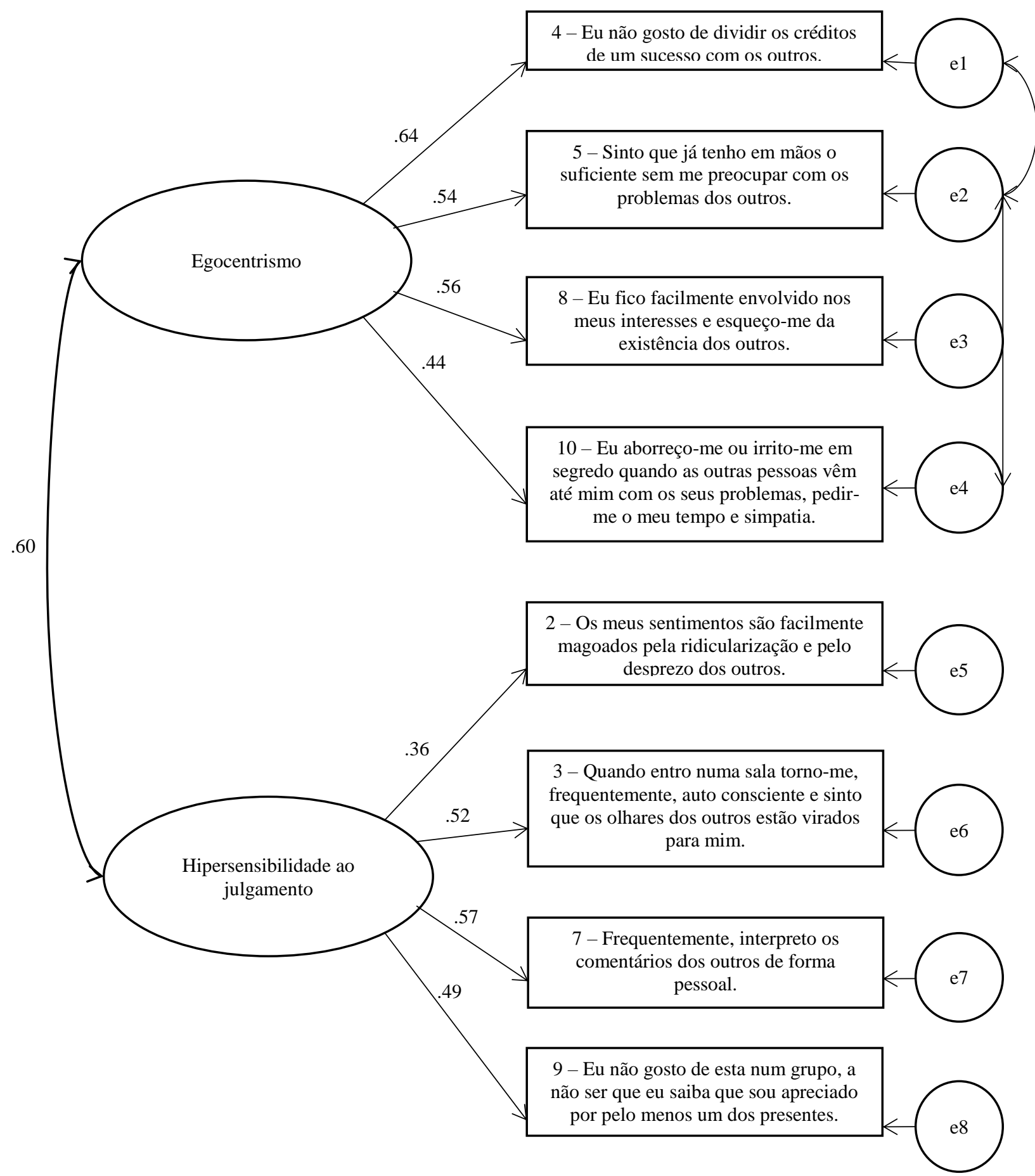

Figura 1- Modelo Bifatorial (final) do HSNS, com respetivos valores dos coeficientes de regressão, proporção da variância explicada pelo preditor e covariâncias entre erros.

Relativamente à proporção de variância dos itens explicada pelos respetivos fatores, os valores são tendencialmente baixos, variando entre $.13 \mathrm{e}$ .42 mas com significância estatística $(p<.001)$ (Tabachnick \& Fidel, 2007). Os dois fatores apresentam uma correlação significativa entre si de $.60(p<.001)$.

Através da presente amostra, o HSNS (modelo unifatorial) apresenta um alfa de Cronbach de .66 e uma média de correlações entre itens de .17. As correlações item-total variam entre .13 e .44 , sendo o item 1 o mais problemático com .13.

O Modelo $2 \mathrm{~F}$ apresenta valores de alfa de Cronbach de .68 para o total (dos 8 itens), .61 para o fator egocentrismo e .54 para o fator hipersensibilidade ao julgamento. 
Quadro 4. Correlações entre o FFM (abertura à experiência, conscienciosidade, extroversão, amabilidade e neuroticismo), o narcisismo vulnerável (HSNS) e o narcisismo grandioso (NPI). (N=273)

\begin{tabular}{|c|c|c|c|c|c|}
\hline \multirow[b]{3}{*}{$\begin{array}{l}\text { Narcisismo vulnerável } \\
\text { (HSNS) }\end{array}$} & \multicolumn{5}{|c|}{ NEO-FFI } \\
\hline & $\begin{array}{l}\text { Abertura à } \\
\text { experiência }\end{array}$ & Conscienciosidade & Extroversão & Amabilidade & Neuroticismo \\
\hline & $-.157^{* *}$ & $-.426^{* *}$ & $-.375^{* *}$ & $-.462^{* *}$ & $.491^{* *}$ \\
\hline $\begin{array}{l}\text { Narcisismo grandioso } \\
\text { (NPI) }\end{array}$ & $.208^{* *}$ & -0.012 & $.325^{* *}$ & $-.139^{*}$ & $-.255^{* *}$ \\
\hline
\end{tabular}

Nota. $* p<.05 ; * * p<.01$.

Os resultados deste estudo remetem assim para uma solução do HSNS conforme a Figura 1, com dois fatores e em que o total e os fatores são calculados através das seguintes fórmulas:

HSNS total $=$ item $4+$ item $5+$ item $8+$ item 10 + item 2 + item 3 + item $7+$ item 9 ;

Fator 1 (egocentrismo) $=($ item $4 \times .255)+$

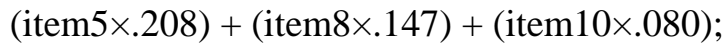

Fator 2 (hipersensibilidade ao julgamento) $=$

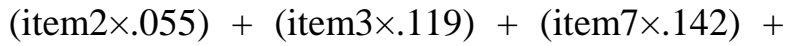
(item9×.083).

Através das correlações entre os cinco fatores da personalidade do FFM (NEO-FFI; abertura à experiência, conscienciosidade, extroversão, amabilidade e neuroticismo), o narcisismo vulnerável (HSNS) e o narcisismo grandioso (NPI) foram obtidos os resultados do Quadro 4.

\section{Discussão}

O modelo final do HSNS obtido para esta amostra da população portuguesa revelou consistência no seu construto através da estrutura bifatorial em que a distribuição dos itens é semelhante à encontrada no estudo de Fossati et al. (2009). O fator 1 (egocentrismo) é composto pelos itens $4,5,8$ e 10 e $\mathrm{o}$ fator 2 (hipersensibilidade ao julgamento) pelos itens 2, 3,7 e 9. O item 6 ("Sinto que sou temperamentalmente diferente da maioria das pessoas.") revelou uma saturação inferior a .32 tendo sido excluído aquando da AFE. Também o item 1 ("Sou capaz de ficar inteiramente absorvido nos meus pensamentos acerca dos meus assuntos pessoais, da minha saúde, das minhas preocupações, ou relações com os outros.") foi excluído da $\mathrm{AFC}$ devido à baixa correlação itemtotal (.13).

Os valores de ajustamento global revelam-se adequados e o modelo evidencia um número reduzido de alterações realizadas para a melhoria do ajustamento. Ao nível do ajustamento local todos os itens demonstram valores de coeficientes de regressão adequados (entre .36 e .64; $p<.001$ ) (Tabachnick \& Fidel, 2007). Os dois fatores apresentam uma correlação significativa entre si de $.60(p<.001)$.

Os valores de alfa de Cronbach estão abaixo do desejável, com .68 para o total (dos 8 itens), .61 para o fator egocentrismo (na versão italiana é de .62) e .54 para o fator hipersensibilidade ao julgamento (.66 na versão italiana; Fossati et al., 2009). No entanto, o número de itens influência o cálculo do alfa (Pires et al., 2018) e a fragilidade do alfa a medidas pequenas tem sido já referida (Pallant, 2005) e o HSNS é uma escala com um número de itens reduzido.

De acordo com o esperado (Hendin \& Cheek, 1997; Houlcroft et al., 2012; Miller et al., 2010a; Miller et al., 2010b; Miller et al., 2011) o narcisismo vulnerável (HSNS) revela uma forte relação positiva com o neuroticismo e relações médias negativas com a extroversão e a amabilidade. Vários autores têm verificado resultados semelhantes, reportando o narcisismo vulnerável associado a baixa extroversão, baixa amabilidade e elevado neuroticismo (Houlcroft et al., 2012; Miller et al., 2010a, Miller \& Campbell, 2008).

Do mesmo modo, de acordo com a literatura (Houlcroft et al., 2012; Mathieu, 2013; Miller \& Campbell, 2008; Paulhus \& Williams, 2002; Miller et al., 2010a; Miller et al., 2010b; Miller et al., 2011), o narcisismo grandioso (NPI) está positivamente relacionado com a extroversão e negativamente relacionado com amabilidade e o neuroticismo.

Como defendem Pincus e Lukowitsky (2010), a importância de estudar os dois padrões de narcisismo de forma diferenciada fica evidente 
perante os perfis que o NPI (NG) e o HSNS (NV) revelaram nas correlações com a personalidade (NEO-FFI), tendo como única semelhança os baixos níveis de amabilidade, já muito referidos nas investigações anteriores (Miller et al., 2010b; Miller et al., 2011) mas dissociando-se ao nível do neuroticismo e da extroversão.

Estes resultados vão ao encontro daquilo que Hendin e Cheek (1997) referiam, de que o NPI estaria associado a um perfil de narcisismo distinto do HSNS.

Perante os resultados do presente estudo, na linha de outros estudos (Fossati et al., 2009; Hendin \& Cheek, 1997; Ripoll, Salazar e Bobes, 2010), pode considerar-se que o HSNS apresenta potencial para avaliar o narcisismo vulnerável nas diversas populações - ainda que sejam necessárias mais investigações, nomeadamente com outras populações. A diversidade da população mundial sugere uma grande necessidade de realizar investigações interculturais com as medidas para que consigam chegar/medir um número de questões específicas de cada cultura e nacionalidade (Sousa \& Rojjanasrirat, 2011).

Algumas limitações a considerar neste estudo têm que ver com o facto de a amostra ter sido recolhida através de uma rede social e de mais de $50 \%$ dos participantes serem estudantes. Deste modo, serão necessários mais estudos, com amostras que possam ser mais representativas da população em geral. Outra questão importante tem que ver com a versão do NPI utilizada nas comparações com o NEO-FFI (Pereira, 2015). Esta versão apresenta uma estrutura diferente de todas aquelas que foram anteriormente propostas na literatura, dificultando a validade de estudos comparativos/replicativos.

\section{Referências}

Ackerman, R. A., Witt, E. A., Donnellan, M. B., Trzenesniewski, K. H., Robins, R. W., \& Kashy, D. A. (2011). What does the narcissistic personality inventory really measure? Assessment, 18, 67-87.

Arble, E. P. (2008). Evaluating the psychometric properties of the Hypersensitive Narcissism Scale: Implications for the distinction of covert and overt narcissism. Masters Thesis and Doctoral Dissertations, Paper 236.

Basto-Pereira, M., Miranda, A., Ribeiro, S., \& Maia, Â. (2016). Growing up with adversity: From juvenile justice involvement to criminal persistence and psychosocial problems in young adulthood. Child Abuse \& Neglect, 62, 63-75.

Besser, A., \& Zeigler-Hill, V. (2010). The influence of pathological narcissism on emocional and motivational responses to negative events: The roles of visibility and concern about humiliation. Jounal of Research in Personality, 44, 520-534.

Cain, N. M., Pincus, A. L., \& Ansell, E. B. (2008). Narcissism at the crossroads: Phenotypic description of pathological narcissism across clinical theory, social/personality psychology, and psychiatric diagnosis. Clinical Psychology Review, 28, 638-656.

Campbell, K., Rudich, E., \& Sedikides, C. (2002). Narcissism, self-esteem and positivity of selfviews: two protraits of self-love. Society for Personality and Social Psychology, 28, 358368.

Cooper, A. M. (2009). The Narcissisticmasochistic Character. Psychiatric Annals, 39, 904-912.

Corry, N., Merritt, D., Mrug, S., \& Pamp, B. (2008). The Factor Struture of the Narcissistic Personality Inventory. Journal of Personality Assessment, 90, 593-600.

Dickinson, K., \& Pincus, A. (2003). Interpersonal analysis of grandiose and vulnerable narcisssm. Journal of Personality Disorders, 188-207.

Emmons, R. A. (1987). Narcissism: Theory and measurement. Journal of Personality and Social Psychology, 52, 11-17

Fossati, A., Borroni, S., Grazioli, F., Dornetti, L., Marcassoli , I., Maffei , C., \& Cheek, J. (2009). Tracking the hypersensitive dimension in narcissism: Reliability and validity of the Hypersensitive Narcissism Scale. Personality and Mental Health, 3, 235247.

Foster, J. D., \& Campbell, W. K. (2005). Narcissism and resistance to doubts about 
romantic partners. Journal of Research in Personality, 39, 550-557.

Foster, J. D., Campbell, W. K., \& Twenge, J. M. (2003). Individual differences in narcissism: Inflated self-views across the lifespan and around the world. Journal of Research in Personality, 37, 469-486.

Foster, J., \& Campbell, W. (2007). Are there such things as "Narcissists" in social psychology? A taxometric analysis of the Narcissistic Personality Inventory. Personality and Individual Diferences , 1321-1332.

Given-Wilson, Z., Mcllwain, D., \& Warburton, W. (2011). Meta-cognitive and interpersonal difficulties in overt end covert narcissism. Personality and Individual Differences, 50, 1000-1005.

Hendin, H. M., \& Cheek, J. M. (1997). Assessing Hypersensitive Narcissism: A reexamination of Murray's Narcism Scale. Jounal of Research in Personality, 31, 588-599.

Hill, M. M., \& Hill, A. (2005). Investigação por questionário ( $2^{\mathrm{a}}$ ed. rev. corrig., $2^{\mathrm{a}}$ reimp. ed.). Lisboa: Sílabo.

Houlcroft, L., Bore, M., \& Munro, D. (2012). Three faces of narcissism. Personality and Individual Differences, 53, 274-278.

Johnson, J., Cohen, P., Brown, J., Smailes, E., \& Bernstein, D. (1999). Childhood maltreatment increases risk for personality disorders during early adulthood. Archives of General Psychiatry, 56(7), 600-606.

Kealy, D., \& Rasmussen, B. (2012). Veiled and vulnerable: The other side of grandiose narcissism. Clinical Social Work Journal, 40, 356-365.

Kealy, D., Ogrodniczuk, J. S., Joyce, A. S., Steinberg, P. I., \& Piper, W. E. (2013). Narcissism and relational representations among psychiatric outpatients. Journal of Personality Disorders, 27, 1-15.

Kurbarych, T. S., Deary, I. J., \& Austin, E. J. (2004). The narcissistic personality inventory: Factor structure in a nonclinical sample. Personality and Individual Differences, 36, 857-872.

Lee, K., \& Ashton, M. C. (2005). Psychopathy, machiavellianism, and narcissism in the FiveFactor Model and the HEXACO model of personality structure. Personality and Individual Differences, 38, 1571-1582.

Lee, V. \& Hoaken, P. (2007). Cognition, emotion, and neurobiological development: Mediating the relation between maltreatment and aggression. Child Maltreatment, 12(3) 281298.

Luchner, A., Houston , J., Walker, C., \& Houston, M. (2011). Exploring the relationship betwenn two forms of narcissism and competitiveness. Personality and Individual Differences 51, 779-782.

Magalhães, E., Salgueira, A., Gonzalez, A. J., Costa, J. J., Costa, M. J., Costa, P., \& Pedroso-Lima, M. (2014). NEO-FFI: Propriedades psicométricas de um Inventário Reduzido de Personalidade no Contexto Português. Psicologia: Reflexão e Crítica , 27, 642-657.

Mathieu, C. (2013). Personality and job satisfaction: The role of narcissism. Personality and individual differences, 55, 650-654.

Maxwell, K., Donnellan, M. B., Hopwood, C. J., \& Ackerman, R. A. (2011). The two faces of Narcissus? An empirical comparison of the Narcissistic Personality Inventory and the Pathological Narcissism Inventory. Personality and Individual Differences, 50, 577-582.

Miller, J. D., Dir, A., Gentile, B., Wilson, L., Pryor, L. R., \& Campbell, W. K. (2010a). Searching for a vulnerable dark triad: Comparing factor 2 psychopathy, vulnerable narcissism, and borderline personality disorder. Journal of personality, 78, 15291564.

Miller, J. D., Hoffman, B. J., Gaugham, E. T., Gentile, B., Maples, J., \& Campbell, W. K. (2011). Grandiose and vulnerable narcissism: A nomological network analysis. Journal of Personality, 79, 1013 - 1042.

Miller, J. D., Price, J., Gentile, B., Lynam, D. R., \& Campbell, W. K. (2012). Grandiose and vulnerable narcissism from the perspective of the interpersonal circumplex. Personality and individual differences, 53, 507-512.

Miller, J. D., Widiger, T. A., \& Campbell, W. K. (2010b). Narcissistic personality disorder and 
DSM-V. Journal of Abnormal Psychology, 119, 640-649.

Miller, J., \& Campbell, W. (2008). Comparing clinical and social-personality conceptualizations of narcissism. Jornal of Personality, 76, 449-476.

Morf, C., \& Rhodewalt, F. (2001). Unraveling the paradoxes of narcissism: A dynamic selfregulatory processing model. Psychological Inquiry, 12, 177-196.

Pallant, J. (2005). SPSS Survival manual. A step by step guide to data analysis using SPSS for Windows (Version 12) (2nd edition ed.). Australia: Allen \& Unwin.

Paulhus, D. L. \& Williams, K. M. (2002). The Dark Triad os Personality: Narcissism, Machiavellianism and psychopathy. Journal of Research in Personality, 36, 556-563.

Pechorro, P., Gentile, B., Ray, J., Nunes, C., \& Gonçalves, R. (2016). Adaptation of the narcissistic personality inventory among a portuguese sample of incarcerated juvenile offenders. Psychology, Crime \& Law, 22(5), 495-511.

Pechorro, P., Maroco, J., Ray, J., Gonçaves, R., \& Nunes, C. (2018). A brief measure of narcissism among female juvenile delinquents and community youths: the narcissistic personality inventory-13. International Journal of Offender Therapy and Comparative Criminology, 62, 2292-2311.

Pedroso-Lima, Margarida, Magalhães, Eunice, Salgueira, Ana, Gonzalez, António-José, Costa, José Joaquim, Costa, Manuel João, \& Costa, Patrício. (2014). A versão portuguesa do NEO-FFI: Caracterização em função da idade, género e escolaridade. Psicologia, 28(2), 01-10.

Pereira, C. (2015), Satisfação sexual, personalidade e narcisismo (Tese de Mestrado). Disponível no repositório digital da Universidade de Coimbra. URL:http://hdl.handle.net/130316/30970.

Pincus, A., \& Lukowitsky, M. (2010). Pathological narcissism and narcissitic personality disorder. Annual Review of Clinical Psychology, 6, 421-446.

Pires, R., Ferreira, A., Guedes, D., Gonçalvez, B., \& Henriques-Calado, J. (2018). Estudo das propriedades psicométricas - Formas Longa,
Reduzida e Breve - da Versão Portuguesa do Inventário da Personalidade para o DSM-5 (PID-5). Revista Iberoamericana de Diagnóstico y Evaluación - e Avaliação Psicológica. 47(2), 197-212.

Prieto, G., Muniz, J., Almeida, S., \& Bartram, D. (1999). Uso de los tests psicológicos en España, Portugal e Iberoamérica. Revista Iberoamericana de Diagnóstico y Evaluación - e Avaliação Psicológica. 2(8), 67-82.

Raskin, R., \& Terry, H. (1988). A principalcomponents analysis of the Narcissistic Personality Inventory and further evidence of its construct validity. Journal of Personality and Social Psychology, 54, 890-902.

Ripoll, C., Salazar, J., \& Bobes, J. (2010). Validez de la versión española de la Hypersensitive Narcissism Scale (HSNS) en una Unidad de Conductas Adictivas. Adicciones, 22, 29-36.

Roche, M., Pincus, A., Lukowitsky, M., Ménard, K., \& Conroy, D. (2013). An integrative approach to the assessment of narcissism. Journal of Personality Assessment, 95 , 273248.

Ronningstam, E. (2009). Narcissistic personality disorder: Facing DSM-V. Psychiatric annals, 39(3), $111-121$.

Ryan, K., Weikel, K., \& Sprechini, G. (2008) Gender differences in narcissism and courtship violence in dating couples. Sex Roles, 58, 802-813.

Sousa, V. \& Rojjanasrirat, W. (2011). Translation, adaptation and validation of instruments os scales for use in cross-cultural health care research: A clear and user-friendly guideline. Journal of Evaluation in Clinical Practice, 17, 268-274.

Tabachnick, B. G., \& Fidell, L. S. (2007). Using multivariate statistics (5th edition ed.). Boston: Pearson/Allyn \& Bacon.

Thomaes, S., Bushman, B., Castro, B., \& Stegge, H. (2009). What makes narcissists bloom? A framework for research on the etiology and development of narcissism. Development and Psychopathology, 21, 1233-1247.

Tritt, S. M., Ryder, A. G., Ring, A. J., \& Pincus, A. L. (2010). Pathological narcissism and the depressive temperament. Journal of Affective Disorders, 122, 280-284. 
Twenge, J., Konrath, S., Foster, J., Campbell, W., \& Bushman, B. (2017). Egos inflating over time: A cross-temporal meta-analysis of the narcissistic personality inventory. Journal of Personality, 76(4), 875-901.

Wink, P. (1996). Narcissism. Em C. G. Costello, Personality characteristics of the personality disordered (146-172). New York, New York: Wiley.

Zeigler-Hill, V., Green, B., Arnau, R., Sisemore, T., \& Myers, E. (2011). Trouble ahead, trouble behind: Narcissism and early maladaptative schemas. Journal of Behavior Therapy and Experimental Psychiatry, 42, 96103. 